\title{
Primary Breast Lymphoma: A Single-institute Experience in Taiwan
}

\author{
Che-Wei Ou ${ }^{1}$, Lee-Yung Shih ${ }^{1,2}$, Po-Nan Wang ${ }^{1}$, Hung Chang ${ }^{1}$, Ming-Chung Kuo ${ }^{1}$, Tzung-Chih Tang ${ }^{1}$, \\ Jin-Hou Wu ${ }^{1}$, Tung-Liang Lin ${ }^{1}$, Yu-Shin Hung ${ }^{1}$, Po Dunn ${ }^{1,2}$
}

Background: Breast is an uncommon location of lymphoma involvement. The most common type of primary breast lymphoma (PBL) is diffuse large B-cell lymphoma (DLBCL). Rituximab is the widely used monoclonal antibody against CD20+ B-cell lymphoma, especially DLBCL. We aimed to analyze the clinical features, prognostic factors, and treatment outcome with or without rituximab in primary breast DLBCL.

Methods: We retrospectively analyzed patients diagnosed with PBL from October 1987 to March 2012 in our hospital, excluding metastasis by whole-body computed tomography and bone marrow study.

Results: $\quad$ Twenty-three patients were diagnosed with PBL. All were females. Eighteen patients were stage IE and five were stage IIE according to the Ann Arbor staging system. Two patients had lymphoma other than DLBCL. The median age of primary breast DLBCL patients was 48 years (range 27-79). Two were excluded from the analysis due to refusal or ineligibility for chemotherapy. No significant prognostic factor was found. Patients receiving chemotherapy with $(\mathrm{RC})$ or without $(\mathrm{C})$ rituximab were not significantly different in the 5 -year overall

\begin{abstract}
At a Glance Commentary
Scientific background of the subject

Primary breast lymphoma is rare. This study aimed to analyze clinical features of patients with primary breast lymphoma in Chang Gung Memorial Hospital at Linkou, search for prognostic factors and relapse pattern in patients with primary breast diffuse large B-cell lymphoma, and compare treatment outcome between patients receiving rituximab and those not receiveing rituximab.
\end{abstract}

\section{What this study adds to the field}

According to our study, patients seemed to be younger in Taiwanese cohorts. No prognostic factor was found. Relapse in the central nervous system was not uncommon. Addition of rituximab to chemotherapy did not bring about significant benefits. survival (RC: $57.1 \%$; C: $58.3 \%$; $p=0.457$ ) or progression-free survival (RC: $57.1 \%$; C: $50.0 \%$; $p=0.456)$. A high incidence of relapse in the central nervous system (CNS) (17.6\%) was observed.

Conclusions: In accordance with prior literature reports, our Taiwanese cohort of primary breast DLBCL seemed younger than those reported in Japan, Korea, and Western societies. Relapse in the CNS was not uncommon. The benefit of rituximab in addition to chemotherapy was not statistically significant. Treatment modality remained to be defined by further large-scale studies. (Biomed J 2014;37:321-325)

Key words: diffuse large B-cell lymphoma, primary breast lymphoma, rituximab

$\mathrm{P}$ rimary breast lymphoma (PBL) is rare and accounts for $0.38-0.7 \%$ of non-Hodgkin's lymphoma (NHL). ${ }^{[1-3]}$ The most common type of pathology of PBL is diffuse large B-cell lymphoma (DLBCL). The optimal treatment of PBL remains undefined..$^{[1,3-10]}$ On the other side, the use of rituximab has brought a new era in the treatment of lymphoma. In combination with chemotherapy, rituximab has well-proved efficacy in treatment of DLBCL. ${ }^{[1-15]}$ The

From the ${ }^{1}$ Division of Hematology, Department of Internal Medicine, Chang Gung Memorial Hospital at Linkou, Chang Gung University College of Medicine, Taoyuan, Taiwan; ${ }^{2}$ School of Medicine, Chang Gung University, Taoyuan, Taiwan

Received: May. 03, 2013; Accepted: Sep. 22, 2013

Correspondence to: Dr. Po Dunn, Division of Hematology, Department of Internal Medicine, Chang Gung Memorial Hospital at Linkou. 5, Fusing St., Gueishan Township, Taoyuan County 333, Taiwan (R.O.C.). Tel: 886-3-3281200 ext. 2524; Fax: 886-3-3286697; E-mail: dunnpo@adm.cgmh.org.tw

DOI: $10.4103 / 2319-4170.132889$ 
therapeutic benefit, however, has not been shown for PBL. In Taiwan, rituximab has been prescribed as a part of first-line treatment for DLBCL since 2006. We aimed to analyze the clinical features of the patients diagnosed with PBL in our hospital, and investigate whether the outcome for primary breast DLBCL could be improved by the addition of rituximab.

\section{METHODS}

\section{Patient selection and clinical characteristics}

From October 1987 to March 2012, patients with pathologically proved malignant lymphoma in the breast were selected from the Cancer Registry of Chang Gung Memorial Hospital. The criteria of PBL proposed by Wiseman et al. ${ }^{[16]}$ were adopted, including (1) adequate pathologic evaluation, (2) close association of mammary tissue and lymphomatous infiltration, (3) no evidence of concurrent widespread disease, and (4) no prior extramammary lymphoma. Those who did not receive staging workup or treatment or did not meet the criteria were excluded. Besides, the definition of regional lymph node involvement was strictly limited to the ipsilateral axillary group. The clinical features, pathology, treatment modalities, and outcome were reviewed from medical records.

\section{Statistical analysis}

Chi-square analysis and Fisher's exact test were used for analysis of response rate and relapse rate, respectively. Five-year overall survival (OS) and progression-free survival (PFS) were estimated by the Kaplan-Meier method. Comparison of survival curves was done by the log-rank test. A two-tailed $p$ value less than 0.05 was considered to be significant. All calculations were carried out by using PASW Statistics software system (Windows version 18.0).

\section{RESULTS}

\section{Clinical features and pathology}

There were 23 patients diagnosed with PBL, with the incidence rate of $0.49 \%$ of all NHL patients in a period of 25 years. All patients were females. The initial presentation symptoms were breast mass in all patients except one who initially presented with enlarged ipsilateral axillary lymph nodes. The pathologic types included DLBCL $(n=21$, $91.3 \%)$, B-lymphoblastic lymphoma $(n=1,4.3 \%)$, and unspecified malignant lymphoma due to inadequate pathologic specimen for classification $(n=1,4.3 \%)$. Median follow-up time was 45 months (range 5-241). The median age of patients with DLBCL was 48 years (range 27-79).
One was unsuitable for chemotherapy due to pneumonia after operation, and she died shortly after diagnosis. Another one refused chemotherapy for personal reasons. The remaining 19 patients received systemic chemotherapy composed of cyclophosphamide, adriamycin, vincristine, and prednisolone (CHOP) or CHOP-like regimen with or without rituximab. The clinical characteristics are listed in Table 1. In brief, left and right breasts were equally involved, and none had bilateral involvement. Most patients had good performance status and normal lactate dehydrogenase (LDH). All patients had low risk by International Prognostic Index (IPI). Only one patient had B symptoms.

\section{Treatment modalities and outcome}

All patients included in the analysis received systemic chemotherapy. Three patients had received mastectomy before chemotherapy. One patient had chemotherapy followed by local radiotherapy for her bulky disease. Seven patients received rituximab in addition to systemic chemotherapy. The remaining 12 patients with DLBCL received chemotherapy alone. Cycles of chemotherapy ranged from 5 to 8 . In these 19 patients, 17 (89.5\%) achieved complete remission. No maintenance therapy was prescribed once patients had achieved complete remission. After patients achieved complete remission, they were followed up regularly with physical examinations and blood tests, including hemogram and biochemistry, every 2-3 months. Besides, whole-body computed tomography from brain to pelvis was performed every 6 months in the first 2 years. Once PFS was longer than 2 years, image study was performed only when relapse was suspected. One patient receiving chemotherapy alone had progressive disease, and the other patient receiving rituximab in addition to chemotherapy had only partial response. Correlation between the remission and relapse status and the addition of rituximab is shown in Table 2. Adding rituximab did not affect the rate of complete response (6/9 vs. $11 / 18, p=1.0)$. Of the 17 patients who had initial complete response, 8 (47.1\%) suffered from relapse. Adding rituximab did not affect the incidence of relapse, either $(2 / 6$ vs. $6 / 11, p=0.62)$.

In the patients with relapse, none had relapse at the primary site. Two patients had relapse in the contralateral breast, and one of them had concurrent relapse in the central nervous system (CNS). The remaining six patients had relapse in remote areas, including the CNS $(n=2)$ and distant lymph node regions $(n=4)$. Relapse rate of the CNS was $17.6 \%$ (3/17). Six relapsed patients received salvage chemotherapy. Intrathecal chemotherapy was also prescribed for the patients with CNS relapse. Only two patients who relapsed at distant lymph nodes achieved second remission, including the one receiving subsequent stem cell transplantation. Although both patients had remission, neither of 
Table 1: Clinical features of 21 patients with primary breast DLBCL

\begin{tabular}{|c|c|c|}
\hline Variables & No. & $\%$ \\
\hline \multicolumn{3}{|c|}{ Age, years median 48 (27-79) } \\
\hline$>60$ & 5 & 23.8 \\
\hline$<60$ & 16 & 76.2 \\
\hline \multicolumn{3}{|l|}{ Performance status } \\
\hline 0 & 13 & 61.9 \\
\hline 1 & 7 & 33.3 \\
\hline$>1$ & 1 & 4.8 \\
\hline \multicolumn{3}{|l|}{ Laterality } \\
\hline Right & 11 & 52.4 \\
\hline Left & 10 & 47.6 \\
\hline \multicolumn{3}{|l|}{ Stage } \\
\hline IE & 16 & 76.2 \\
\hline IIE & 5 & 23.8 \\
\hline \multicolumn{3}{|l|}{ B symptoms } \\
\hline Present & 1 & 4.8 \\
\hline Absent & 20 & 95.2 \\
\hline \multicolumn{3}{|l|}{ LDH } \\
\hline Normal & 20 & 95.2 \\
\hline Elevated & 0 & 0 \\
\hline Unknown & 1 & 4.8 \\
\hline \multicolumn{3}{|l|}{ IPI } \\
\hline 0 & 14 & 66.7 \\
\hline 1 & 7 & 33.3 \\
\hline \multicolumn{3}{|l|}{ Treatment } \\
\hline Mastectomy & 3 & 14.3 \\
\hline Radiotherapy & 1 & 4.8 \\
\hline Chemotherapy & 19 & 90.5 \\
\hline Chemotherapy+R & 7 & 33.3 \\
\hline
\end{tabular}

Abbreviations: BLL: B-lymphoblastic lymphoma; R: Rituximab; DLBCL: Diffuse large B-cell lymphoma; LDH: Lactate dehydrogenase; IPI: International prognostic index

Table 2: Treatment response and outcome of patients with primary breast DLBCL

\begin{tabular}{lcccc}
\hline $\begin{array}{l}\text { Response } \\
\text { No. }(\%)\end{array}$ & $\begin{array}{c}\text { Stage I } \\
\text { with R } \\
(\%)\end{array}$ & $\begin{array}{c}\text { Stage II } \\
\text { with R } \\
(\%)\end{array}$ & $\begin{array}{c}\text { Stage I } \\
\text { without R } \\
(\%)\end{array}$ & $\begin{array}{c}\text { Stage II } \\
\text { without R } \\
(\%)\end{array}$ \\
\hline Complete remission & $4(66.7)$ & $2(66.7)$ & $10(58.8)$ & $1(100)$ \\
Partial remission & 0 & $1(33.3)$ & 0 & 0 \\
Progressive disease & 0 & 0 & $1(5.9)$ & 0 \\
Relapse & $2(33.3)$ & 0 & $6(35.3)$ & 0 \\
\hline
\end{tabular}

All patients received chemotherapy with $\mathrm{CHOP}$ or $\mathrm{CHOP}-$ like regimen. Abbreviations: R: Rituximab; DLBCL: Diffuse large B-cell lymphoma

them survived. One died of pneumonia and the other died of sepsis. All of the patients with refractory disease died.

\section{Prognostic factors and survival curves}

The prognostic factors were corrected with OS and PFS. With univariate analysis, we found there was no significant difference in 5-year OS and PFS associated with clinical variables including age (60 years), stage, performance status, or IPI. We analyzed 5-year OS and PFS between patients receiving chemotherapy plus rituximab or not. Five-year $\mathrm{OS}$ of patients receiving chemotherapy with rituximab (RC) or without (C) was insignificant (RC: $57.1 \%$; C: $58.3 \%$; $p=0.457$ ). Five-year PFS also was insignificant (RC: $57.1 \%$; C: $50.0 \% ; p=0.456$ ). Five-year OS and PFS of these treated patients were $57.9 \%$ and $52.6 \%$, respectively.

\section{DISCUSSION}

Although the incidence of PBL differed between various studies, PBL was clearly a rare subtype of extranodal lymphoma which accounted for $0.38-0.7 \%$ of all forms of NHL. ${ }^{[1,3,17,18]}$ Most studies used the definition of PBL proposed by Wiseman et al. Besides, Wiseman et al. considered that simultaneous ipsilateral axillary lymph node involvement was allowed for the definition of PBL. However, the definition of widespread disease was somewhat controversial if stage workup was made according to the Ann Arbor system, ${ }^{[19]}$ especially for the ipsilateral supraclavicular lymph nodes. As different definitions of PBL were adopted by various studies, ${ }^{[1,3-5,7,18]}$ this potentially would affect the incidence or even outcome analysis results. In our study, we strictly defined PBL as breast lesion with or without concurrent involvement of ipsilateral axillary lymph node. Using such definition, PBL accounts for $0.49 \%$ of all forms of NHL in our cohort, which is similar to that reported in other studies adopting the same criteria for PBL. ${ }^{[18]}$

Regarding laterality, although the largest study of PBL so far showed predilection of right breast involvement, as in ours, left side predilection has been reported and there was no obvious tendency of laterality reported in most other studies. ${ }^{[4,20-22]}$ The reason for such differences is still unknown, and the evidence of laterality predilection is limited.

In our study, the median age of 48 years appears relatively less compared with the age reported in other studies..$^{[1,3,4,7,17,18,23]}$ However, in line with the present study, there are studies reporting patients' age comparable to our cohort. ${ }^{[5,8,21,24]}$ Some scattered reports suggested that younger patients more often had breast Burkitt's lymphoma which tended to involve bilateral breasts. ${ }^{[16,25]}$ Such a relationship of age, laterality, and Burkitt's lymphoma was not observed in other reports. ${ }^{[5,8,21,24]}$ No Burkitt's lymphoma was diagnosed in our patients, which possibly was due to the limited patient number in our study or due to differently low incidence in our patient cohort. Interestingly, except a French cohort study reported by Gholam et al., with a median age of 50, all other studies reporting PBL with younger patient age have been reports of Taiwanese and Chinese patients. ${ }^{[5,8,21,24]}$

Distribution of the pathologic type was another feature that distinguished the present study from earlier ones. Although DLBCL was the most common type of PBL, the 
frequency of DLBCL ranged from only 40 to $80 \%$ in Western reports. ${ }^{[26-28]}$ However, DLBCL accounted for the greater majority of PBL in Taiwanese and Chinese cohorts, ranging from 82.2 to $93.8 \% .^{[5,21,24]}$ Whether such disparities of age and pathologic type resulted from regional or ethnic factors is not clear and futher epidemiological studies are warranted.

Various clinical factors were proposed for prognostic evaluation of PBL, including age, stage, performance status, or serum LDH level..$^{[1,-5,29]}$ According to a review by Caon et al., stage, performance status, and IPI were the most relevant prognostic factors among different studies, of which the patient number in most studies was small and pathologic types were heterogeneous. ${ }^{[29]}$ Therefore, the prognostic impact of such factors was inconsistent. In our study, no clinical variable, such as age, stage, and performance status, was found to be associated with the outcome endpoints of 5-year OS or PFS. As in many other studies, our study was limited by its small scale and retrospective nature. ${ }^{[30]}$ However, for all extranodal DLBCL with limited stage comparable to this patient group, the 5-year OS and PFS were $73 \%$ and $42 \%$, respectively, with anthracycline-containing chemotherapy in our hospital (unpublished data). Although PFS (52.6\%) of primary breast DLBCL was a little better, the OS (57.9\%) was markedly inferior to the limited stage of extranodal DLBCL as a whole. Patients diagnosed with primary breast DLBCL seemed to be associated with a poor prognosis.

The standard treatment modalities have not been established so far. Surgical intervention, chemotherapy, and radiotherapy had been evaluated in many studies. ${ }^{[1,4,8,17,18,31-33]}$ Mastectomy was considered to be of no benefit. ${ }^{[8,18]}$ Some studies reported that anthracycline-containing chemotherapy with radiotherapy provided better outcome than other modalities, but whether such treatment is optimal remains to be validated by large-scale studies. ${ }^{[3,4,24,34]}$ Although rituximab had demonstrated great improvement in the outcome of patients with DLBCL in other anatomic sites, limited studies have shown controversial advancement in the outcome of primary breast DLBCL. ${ }^{[6,7,11-15,34]}$ For example, Yhim et al., reported the addition of rituximab to chemotherapy led to non-inferior outcome in patients with primary breast DLBCL compared with patients with nodal DLBCL. ${ }^{[7]}$ However, Avilés et al. reported that addition of rituximab resulted in no improvement of treatment outcome in patients with primary breast DLBCL in 2007 and 2012, respectively (one prospective and the other retrospective). ${ }^{[6,34]}$

Our response rate to initial treatment was not inferior compared with other studies in which chemotherapy plus radiotherapy was the first-line treatment. ${ }^{[4,5,24]}$ Five-year OS was a little inferior compared with most studies (53-84\%), but PFS was similar (41-50\%)..$^{[1,3-5,8]}$ The difference may be attributed to several reasons. Our cohort had higher frequency of DLBCL compared with the reports of Western society. ${ }^{[1,3,4,8]}$ Once focusing on the outcome of primary breast DLBCL patients, our study had similar 5-year OS in comparison with the largest study so far by International Extranodal Lymphoma Study Group. ${ }^{[4]}$ In addition, we observed a higher CNS relapse rate compared with another report of a Taiwanese cohort. ${ }^{[5]}$

Although most of our primary breast DLBCL patients achieved complete remission after initial treatment, relapse was common with a frequency of $47.1 \%$, which was as high as other reports, especially in the CNS. ${ }^{[1,3,35,36]}$ The CNS relapse rate was variable among studies, leading to a controversial conclusion about CNS prophylaxis in PBL patients. ${ }^{[1,4,5,16,26,28,37]}$ Furthermore, second primary CNS lymphoma was once reported, meaning that real CNS relapse rate is actually uncertain. ${ }^{[5]}$ To determine definite relapse of PBL, more detailed examination such as clonality and gene expression patterns might be needed. On the other hand, our patients did not have local relapse even without radiotherapy, whereas systemic relapse was the main relapse pattern. Such a result suggests that addition of radiotherapy for local control might not be so necessary and had controversial benefit for primary breast DLBCL patients.

In the present cohort, Taiwanese patients with primary breast DLBCL appeared to be younger in age. Our patient number was limited, and we did not find any clinical variable as a significant prognostic factor for 5-year OS or PFS in primary breast DLBCL patients. No improvement of outcome after addition of rituximab to chemotherapy was noted, either. High relapse rate, especially in the CNS, remained as a critical issue, and CNS prophylaxis might be warranted. Proper treatment for primary breast DLBCL still needs to be defined in a large collaborative study.

\section{REFERENCES}

1. Wong WW, Schild SE, Halyard MY, Schomberg PJ. Primary non-hodgkin lymphoma of the breast: The Mayo Clinic Experience. J Surg Oncol 2002;80:19-25.

2. Topalovski M, Crisan D, Mattson JC. Lymphoma of the breast. Arch Pathol Lab Med 1999;123:1208-18.

3. Jeanneret-Sozzi W, Taghian A, Epelbaum R, Poortmans P, Zwahlen D, Amsler B, et al. Primary breast lymphoma: Patient profile, outcome and prognostic factors. A multicentre rare cancer network study. BMC Cancer 2008;8:86.

4. Ryan G, Martinelli G, Kuper-Hommel M, Tsang R, Pruneri G, Yuen $\mathrm{K}$, et al. Primary diffuse large b-cell lymphoma of the breast: Prognostic factors and outcomes of a study by the international extranodal lymphoma study group. Ann Oncol 2008;19:233-41.

5. Lin YC, Tsai CH, Wu JS, Huang CS, Kuo SH, Lin CW, et al. Clinicopathologic features and treatment outcome of non-hodgkin lymphoma of the breast: A review of 42 primary and secondary cases in taiwanese patients. Leuk Lymphoma 2009;50:918-24.

6. Avilés A, Castañeda C, Neri N, Cleto S, Jesús Nambo M. Rituximab and dose dense chemotherapy in primary breast lymphoma. Haematologica 2007;92:1147-8. 
7. Yhim HY, Kim JS, Kang HJ, Kim SJ, Kim WS, Choi CW, et al. Matched-pair analysis comparing the outcomes of primary breast and nodal diffuse large b-cell lymphoma in patients treated with rituximab plus chemotherapy. Int J Cancer 2012;131:235-43.

8. Gholam D, Bibeau F, El Weshi A, Bosq J, Ribrag V. Primary breast lymphoma. Leuk Lymphoma 2003;44:1173-8.

9. Mouna B, Saber B, Tijani E, Hind Mr, Amina T, Hassan E. Primary malignant non-hodgkin's lymphoma of the breast: A study of seven cases and literature review. World J Surg Oncol 2012;10:151-6.

10. Park YH. What is the best treatment for primary breast lymphoma? Combined modality should be adapted according to the risk stratification. Leuk Lymphoma 2006;47:2011-2.

11. Feugier P, Van Hoof A, Sebban C, Solal-Celigny P, Bouabdallah R, Fermé $\mathrm{C}$, et al. Long-term results of the r-chop study in the treatment of elderly patients with diffuse large b-cell lymphoma: A study by the groupe d'etude des lymphomes de 1'adulte. J Clin Oncol 2005;23:4117-26.

12. Pfreundschuh M, Kuhnt E, Trümper L, Österborg A, Trneny M, Shepherd L, et al. Chop-like chemotherapy with or without rituximab in young patients with good-prognosis diffuse large-b-cell lymphoma: 6-year results of an open-label randomised study of the mabthera international trial (mint) group. Lancet Oncol 2011;12:1013-22.

13. Coiffier B, Lepage E, Brière J, Herbrecht R, Tilly H, Bouabdallah R, et al. Chop chemotherapy plus rituximab compared with chop alone in elderly patients with diffuse large-b-cell lymphoma. N Engl J Med 2002;346:235-42.

14. Habermann TM, Weller EA, Morrison VA, Gascoyne RD, Cassileth PA, Cohn JB, et al. Rituximab-chop versus chop alone or with maintenance rituximab in older patients with diffuse large b-cell lymphoma. J Clin Oncol 2006;24:3121-7.

15. Sehn LH, Donaldson J, Chhanabhai M, Fitzgerald C, Gill K, Klasa $\mathrm{R}$, et al. Introduction of combined chop plus rituximab therapy dramatically improved outcome of diffuse large b-cell lymphoma in British Columbia. J Clin Oncol 2005;23:5027-33.

16. Wiseman C, Liao K. Primary lymphoma of the breast. Cancer 1972;29:1705-12.

17. Nicola A, Alessandro S, Roberto C, Giovanni B, Stefano T. Primary breast lymphomas: A multicentric experience. World J Surg Oncol 2010;8:53-6.

18. Domchek SM, Hecht JL, Fleming MD, Pinkus GS, Canellos GP. Lymphomas of the breast. Cancer 2002;94:6-13.

19. Carbone PP, Kaplan HS, Musshoff K, Smithers DW, Tubiana M. Report of the committee on hodgkin's disease staging classification. Cancer Res 1971;31:1860-1.

20. Cabras M, Amichetti M, Nagliati M, Orru P, Mamusa A, Angelucci E. Primary non-hodgkin's lymphoma of the breast: A report of 11 cases. Haematologica 2004;89:1527-8.
21. Guo HY, Zhao XM, Li J, Hu XC. Primary non-hodgkin's lymphoma of the breast: Eight-year follow-up experience. Int J Hematol $2008 ; 87: 491-7$

22. Yoshida S, Nakamura N, Sasaki Y, Yoshida S, Yasuda M, Sagara H, et al. Primary breast diffuse large b-cell lymphoma shows a non-germinal center b-cell phenotype. Mod Pathol 2004;18:398-405.

23. Fukuhara S, Watanabe T, Munakata W, Mori M, Maruyama D, Kim SW, et al. Bulky disease has an impact on outcomes in primary diffuse large b-cell lymphoma of the breast: A retrospective analysis at a single institution. Eur J Haematol 2011;87:434-40.

24. Lin Y, Guo XM, Shen KW, Wang JL, Jiang GL. Primary breast lymphoma: Long-term treatment outcomeand prognosis. Leuk Lymphoma 2006;47:2102-9.

25. Brogi E, Harris N. Lymphomas of the breast: Pathology and clinical behavior. Semin Oncol 1999;26:357-64.

26. Hugh JC, Jackson FI, Hanson J, Poppema S. Primary breast lymphoma: An immunohistologic study of 20 new cases. Cancer 1990;66:2602-11.

27. Giardini R, Piccolo C, Rilke F. Primary non-hodgkin's lymphomas of the female breast. Cancer 1992;69:725-35.

28. Ha C, Dubey P, Goyal L. Localized primary non- hodgkin's lymphoma of the breast. Am J Clin Oncol 1998;21:376-80.

29. Caon J, Wai ES, Hart J, Alexander C, Truong PT, Sehn LH, et al. Treatment and outcomes of primary breast lymphoma. Clin Breast Cancer 2012;12:412-9.

30. Fruchart C, Denoux Y, Chasle J, Peny A, Boute V, Ollivier J, et al. High grade primary breast lymphoma: Is it a different clinical entity? Breast Cancer Res Treat 2005;93:191-8.

31. Ganjoo K, Advani R, Mariappan M, McMillan A, Horning S Non-hodgkin lymphoma of the breast. Cancer 2007;110:25-30.

32. el-Ghazawy IM, Singletary SE. Surgical management of primary lymphoma of the breast. Ann Surg 1991;214:724-6.

33. Jennings W, Baker R, Murray S, Howard C, Parker D, Peabody L, et al. Primary breast lymphoma: The role of mastectomy and the importance of lymph node status. Ann Surg 2007;245:784-9.

34. Aviles A, Neri N, Nambo MJ. The role of genotype in 104 cases of diffuse large b-cell lymphoma primary of breast. Am J Clin Oncol 2012;35:126-9

35. Avilés A, Delgado S, Nambo MJ, Neri N, Murillo E, Cleto S. Primary breast lymphoma: Results of a controlled clinical trial. Oncology 2005;69:256-60.

36. Ribrag V, Bibeau F, El Weshi A, Frayfer J, Fadel C, Cebotaru C, et al. Primary breast lymphoma: A report of 20 cases. Br J Haematol 2001;115:253-6.

37. Dixon J, Lumsden A, Krajewski A, Elton R, Anderson T. Primary lymphoma of the breast. Br J Surg 1987;74:214-7. 